\title{
Midair Balloon Interface: A Soft and Lightweight Midair Object for Proximate Interactions
}

\author{
Takuro Furumoto \\ The University of Tokyo \\ Chiba, Japan \\ furumoto@hapis.k.u-tokyo.ac.jp
}

\author{
Takumi Kasai \\ The University of Tokyo \\ Chiba, Japan \\ kasai@hapis.k.u-tokyo.ac.jp
}

\author{
Masahiro Fujiwara \\ The University of Tokyo \\ Chiba, Japan \\ Masahiro_Fujiwara@ipc.i.u- \\ tokyo.ac.jp
}

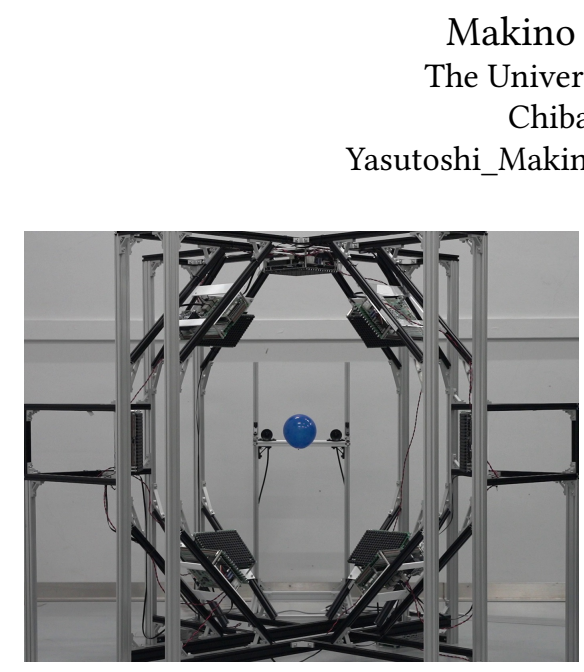
Makino Yasutoshi
Chiba, Japan
Yasutoshi_Makino@k.u-tokyo.ac.jp

Hiroyuki Shinoda

The University of Tokyo

Chiba, Japan

Hiroyuki_Shinoda@k.u-tokyo.ac.jp
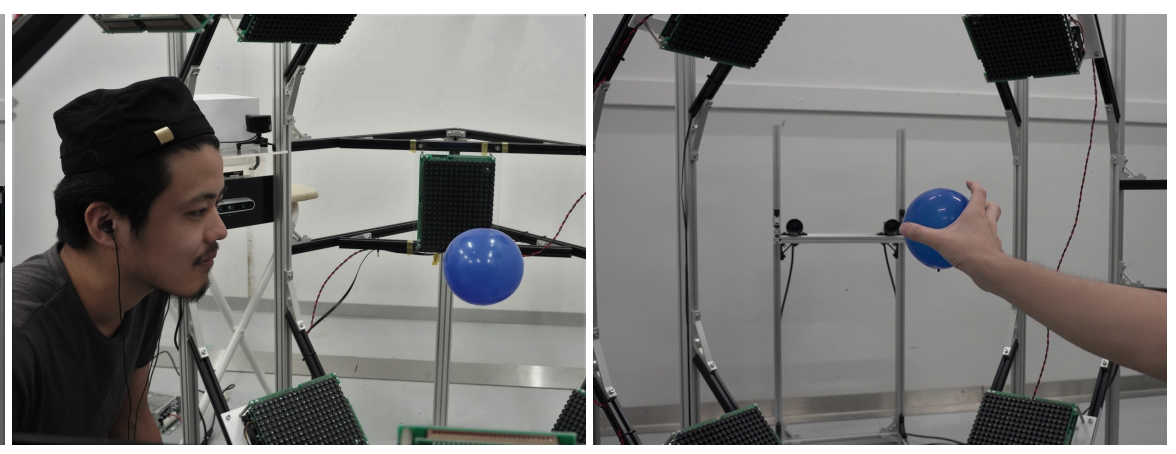

Figure 1: (Left) Prototype system of midair balloon interface. (Center) Balloon object acts as safe-to-collide physical UI, facilitating proximate interactions. (Right) Touch-based interaction allows user to manipulate a balloon object directly with hand and to receive vibrotactile feedback during direct manipulation.

\begin{abstract}
This paper introduces a midair balloon interface, a fast and soft interactive object in mid-air. Our approach tackles the trade-off between safety and speed by controlling a soft helium-filled balloon with external actuators and sensors. We developed a prototype system that uses airborne ultrasound phased arrays to propel a balloon and high-speed stereo cameras to track its motion. This configuration realizes both a high thrust/weight ratio and such a soft body that is safe-to-collide. We describe a sight-based interaction and a touch-based interaction that leverage the safety and speed of a midair balloon interface. A sight-based interaction allows the user to keep the object inside her/his view within reach by controlling a balloon to follow the direction of the user's face. A touch-based interaction allows the user to manipulate the object directly with his/her hand, issue a command by moving his/her finger on the surface, and receive vibrotactile feedback produced by vibrating
\end{abstract}

This work is licensed under a Creative Commons Attribution International 4.0 License.

UIST '21, October 10-14, 2021, Virtual Event, USA

(c) 2021 Copyright held by the owner/author(s).

ACM ISBN 978-1-4503-8635-7/21/10.

https://doi.org/10.1145/3472749.3474786 the balloon with amplitude-modulated ultrasound. We describe the implementation and evaluation of the prototype and explore the application scenarios.

\section{CCS CONCEPTS}

- Human-centered computing $\rightarrow$ Interaction devices; Mixed / augmented reality.

\section{KEYWORDS}

Midair UIs, Soft interfaces, Tangible interfaces, Ultrasound

\section{ACM Reference Format:}

Takuro Furumoto, Takumi Kasai, Masahiro Fujiwara, Makino Yasutoshi, and Hiroyuki Shinoda. 2021. Midair Balloon Interface: A Soft and Lightweight Midair Object for Proximate Interactions. In The 34th Annual ACM Symposium on User Interface Software and Technology (UIST '21), October 10-14, 2021, Virtual Event, USA. ACM, New York, NY, USA, 13 pages. https://doi.org/10.1145/3472749.3474786

\section{INTRODUCTION}

Midair physical objects are emerging components of threedimensional user interfaces (3DUIs). Combined with input/output instruments such as projectors and speakers, they serve as physical UI elements that can be dynamically located in a 3D space. Such midair physical UIs can be seamlessly integrated into the real world 
because users can see and touch them in the same way as with real objects without using special input/output devices or special input modalities. For example, we can use them as a midair "window" by projecting images and texts onto them. We can also use them as midair physical icons (phicons) or controls that can be manipulated directly by hand. Recently, aerial vehicles, or drones, have been increasingly used as midair UIs because they provide a large interaction space and high-speed mobility [11].

Although the use of midair objects is attractive as described above, their safe and comfortable integration into user interface systems is still an open problem, particularly when it involves a direct touch. The best way to ensure safety may be to use hardware that is impossible to harm humans. For example, covering the entire body with soft materials would ensure such safety [34]. This is one of the motivations for developing soft robots for human-robot interactions. Such hardware not only protects users but also appears to be safe, promoting comfortable use within proximity. However, realizing aerial hardware of such safety without sacrificing mobility is challenging.

In this paper, we propose midair balloon interfaces, an approach to realize a safe midair object based on the noncontact actuation of a soft helium-filled balloon, which we refer to as a balloon object. We employ airborne ultrasound phased arrays (AUPAs) to propel a balloon because they can apply force at a distant point without producing perceivable artifacts. Because a balloon object has no rigid parts and consists only of soft and lightweight materials, it has no risk at a collision even at high relative speeds. Furthermore, it has a high thrust/weight ratio, achieving mobility comparable to drone-based interfaces despite the limited actuation force.

We investigate three aspects of midair balloon interfaces. First, we discuss acoustic manipulation of a balloon. Our interest is to provide a balloon object that has a suitable form factor for natural tangible interactions such as grasping and pinching (i.e. several centimeters in length). While many acoustic manipulation techniques exist, they are designed for controlling small particles (< $4.3 \mathrm{~mm}$ diameter), threads or cloth attached to particles, or a large lightweight sphere (e.g. $20 \mathrm{~cm}$-diameter) and are not suitable to the form factor of our interest. In addition, most of them do not allow direct touch to midair objects because direct touch disrupts the acoustic field and may knock them out of the air. To address this, we develop a control method based on sensor feedback stabilization and, more importantly, time-sliced driving of AUPA devices. Acoustic manipulation based on sensor feedback is originally introduced in robotics to control a spherical blimp at low speed (below 200 $\mathrm{mm} / \mathrm{s}$ ). The original method is theoretically limited to large objects (e.g. $20 \mathrm{~cm}$ diameter). We extend the range of the form factor of controllable objects by introducing time-sliced driving of AUPA devices, allowing 3D control of a spherical balloon of any size. The use of a small balloon improves its reachable speed significantly because of its reduced mass and drag force. We evaluate the relationship between the reachable speed and form factor by analyzing the equation of motion that incorporates drag force, which was ignored but has a significant effect on the motion. Then, we demonstrate the 3D control of a balloon of a 10-cm diameter, achieving a peak horizontal speed of $890 \mathrm{~mm} / \mathrm{s}$ and a peak vertical speed of 698 $\mathrm{mm} / \mathrm{s}$, which is four times and three times faster than the previous sensor-feedback-based method respectively.
Next, we discuss interactions with a midair balloon interface. We introduce two interactions that leverage its safety and mobility: sight-based and touch-based interactions. In a sight-based interaction, a balloon object follows the motion of the user's head, keeping itself inside the user's view and within reach. For example, if a balloon interface is used as a midair projection screen, this interaction is useful for presenting information that should be easily notified (e.g., notifications) as well as about the relationship between the user and the environment (e.g., indicating where to look). In addition, if a balloon object is used as a phicon for system control, it is useful to place the control within easy reach. A touch-based interaction allows a user to manipulate a balloon object directly with the user's hand. We report that a midair balloon interface can provide vibrotactile stimulation by vibrating the balloon with amplitude-modulated ultrasound. We utilize this to provide vibrotactile feedback that notifies events during the direct manipulation of a balloon object. This method is different from previous ultrasound midair haptics in that ultrasound is focused on an object in hand rather than directly to the skin, allowing co-location of vibrotactile sensations and passive haptic sensations of a balloon. We investigated the perceived strength of the vibrotactile stimulation method through psychophysical experiments.

Finally, we present four application scenarios that benefit from the safety, mobility, and haptic feedback capabilities provided by midair balloon interfaces.

In summary, we provide the following contributions in this paper:

- We propose the concept of a midair balloon interface.

- We analyze the dynamics of a midair balloon interface and provide a technique that can control a helium-filled spherical balloon of any size.

- We describe the implementation and evaluation of a prototype system that achieves a higher speed than previous sensor-feedback-based systems.

- We propose a vibrotactile stimulation method based on the vibration of a balloon induced by amplitude-modulated ultrasound.

- We evaluated the perceived strength of the proposed vibrotactile stimulation method through psychophysical experiments.

- We explore applications that highlights the advantages of a midair balloon interface.

\section{RELATED WORKS}

\subsection{Interactions with Midair Physical Objects}

The concept of interactions with physical bodies in mid-air appears in various forms such as tangible interfaces [39], programmable matter [38], and Ultimate Display [35]. Previous studies of such interactions can be classified according to the principle of controlling an object in mid-air, which largely determines the interaction properties. We first review interaction systems that use alternative principles and clarify the motivation of this study from a general perspective. Then, we review interactive systems that use ultrasound and discuss the technical differences between existing systems and our study. 
2.1.1 Non-Ultrasound-Based Systems. The initial study provided a midair object by levitating a permanent magnet using the principle of magnetophoresis [22]. The size of the interaction space was a tabletop scale with a height of several centimeters, which is the maximum altitude that can be achieved by magnetophoresis technologies. To scale up the interaction space, we require another principle. Some interface systems employ aerodynamic levitation, achieving vertical movement of up to $300 \mathrm{~mm}$ [3]. Horizontal movement can also be achieved by arranging multiple jets [43].

Recently, aerial vehicles, or drones [11] have gained popularity as aerial platforms for midair tangible interactions. They can provide a large interaction volume and high speed compared to previous methods. The mobility of such vehicles has inspired many types of interactions with midair objects including augmented sports [28], mindfulness [20], dancing [8], telecommunication [44], virtual reality $[1,19]$, and augmented reality $[6,14,42]$.

The higher mobility of hardware has led to increasing attention on safety concerns, particularly when they are operated near humans. Efforts to define standards in terms of physical and psychological aspects are ongoing [2]. One study argued that robots near humans should be entirely covered with soft materials [34]. Typical measures for securing safety are based on covering the fast-moving elements (e.g., rotor blades) and limiting the speed typically to about $0.5 \mathrm{~m} / \mathrm{s}$ [1]. ZeRone attempted to secure safety by employing a blimp-type drone with blade-less propulsion systems [41]. However, current bladeless propulsion systems have a low power-weight ratio, which results in a large form factor and low speed, limiting its application to stationary applications, mainly for visual use. Furthermore, even if it is safe when operating properly, a system failure may cause the fall of a midair rigid object, which may result in injury.

Midair balloon interfaces present a new approach to achieving a safe object by using a midair object that consists of only soft and lightweight materials, disabling it from injuring humans by hardware. This is achieved by the external installation of all actuators. We demonstrate that ultrasound actuation can achieve a speed higher than the current typical regulation at an upper-body scale.

2.1.2 Ultrasound-based Interface Systems. Ultrasound applies force to an object from which it scatters. The force is called the acoustic radiation force. Ultrasound-based UIs utilize this phenomenon to actuate an object without contact.

Most systems use acoustic levitation/manipulation, which is a technology to create a sound field that traps particles that are typically smaller than the half-wavelength of ultrasound in midair [4]. The sound field provides a force against both gravity and disturbances. Their main use is a 3D visualization utilizing levitated particles as voxels [13,30-32], occasionally in combination with grounded objects[9,29]. This group of ultrasound-based interfaces does not include direct haptic interaction with levitated particles because direct touch to the particles disrupts the sound field and may knock off the levitated particles. Therefore, indirect gestures are typically employed as input techniques [10, 30, 32]. For the same reason, haptic experiences are provided by noncontact methods [16]. LeviProps uses levitated particles as anchors that hang acoustically transparent sheets and allows direct contact with the sheet [26].
The other ultrasound-based interfaces use ultrasound only for propulsion. Ultra-tangible moves a ball on an interactive surface as a tangible object [25]. Hopping-pong uses ultrasound to change the trajectory of a ball during ping-pong games [27]. However, 3D control has not been achieved in previous studies.

Our approach is the first midair physical interface based on ultrasound actuation that allows a direct grasp and subsequent manipulation directly with the user's hand in mid-air. In this paper, we analyze the dynamics of the three-dimensional control of a balloon and fully describe the implementation and evaluation of our prototype. Furthermore, we present and evaluate new sight/touchbased input techniques and a vibrotactile feedback method for proximate interactions using a midair balloon object. In contrast to voxel displays that visualize information in mid-air using a set of sparsely aligned particles or a fast-moving particle, our approach provides a continuous interactive surface that has a sufficient area and volume for touching and projecting images.

Technically, our approach belongs to the latter group because the levitation force is mainly provided by helium gas, and only the propulsive force is provided by ultrasound.

\subsection{Midair Ultrasound Haptics}

Ultrasound midair haptics is a technology that utilizes the phenomenon of an acoustic radiation force to provide tactile sensation in mid-air [7, 15, 17, 24]. Focusing sound waves with phased-array transducers can provide tactile stimulation at an arbitrary point and timing without contact. The intensity of the force is on the order of $10 \mathrm{mN}$ and is too small to be perceived as static pressure but is large enough to provide vibrotactile sensations. To be effectively perceived, the ultrasound wave is modulated at a frequency that is sensitive to human vibrotactile sensations.

Previous methods of midair ultrasound haptics provide tactile stimulation by applying ultrasound waves directly to the glabrous skin. One of the difficulties of midair ultrasound haptics is providing tactile sensations to occluded regions of the skin. Our system creates focus on the balloon in the user's hand and excites its vibration instead of directly applying force to the skin. The balloon is used as a medium to deliver vibrations to the skin. This provides vibrotactile sensations that are co-located with the passive haptic sensations provided by the physical body of the balloon, helping users associate the haptic feedback to the balloon they manipulate. In this paper, we confirmed that focusing on a balloon in one's hand can provide vibrotactile stimulation through psychophysical experiments.

\section{OVERVIEW}

Figure 2 shows the overview of our system design of a midair balloon interface. There are two aspects in the creation of the system: the three-dimensional control of a balloon and user interactions.

We use optical tracking sensors and airborne ultrasound phased arrays (AUPAs) to control a balloon in a 3D space. An AUPA can apply acoustic radiation force to a balloon anywhere in mid-air by creating a focus on it. The position of the balloon is measured by using stereo cameras and then used to calculate the driving phases and amplitudes of the AUPAs. Design considerations include the form factor of the balloon, the size, power, and arrangement of AUPAs, an algorithm that computes the driving phases and 


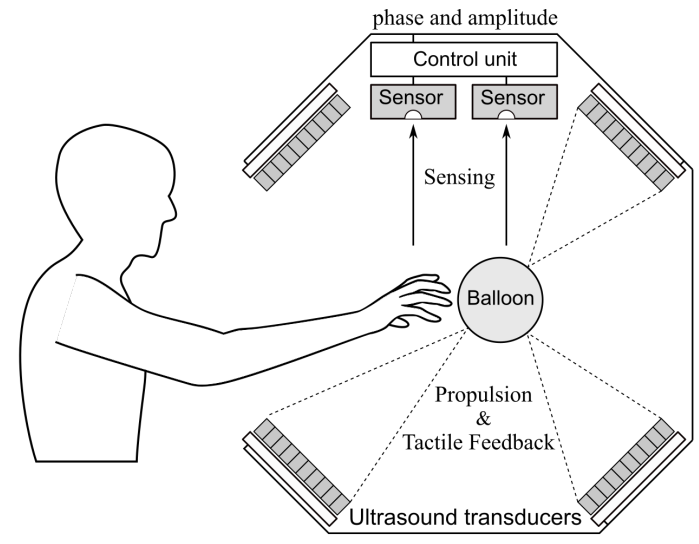

Figure 2: Overview of system design.

amplitudes of transducers, and a hardware implementation that can respond to the speed.

Depth sensors and stereo cameras are used to detect users' actions such as touching, grasping, and clicking. The AUPAs act as off-board haptic actuators when the balloon is in hand. They can produce perceivable vibrations of the balloon by focusing an ultrasound with amplitude modulation at a frequency that is sensitive to cutaneous mechanoreceptors.

\section{THREE-DIMENSIONAL CONTROL OF A BALLOON}

\subsection{Analysing Dynamics}

In this section, we investigate the relationship among actuation force, form factor, and reachable speed. We first clarify the dynamics by solving the equation of motion. Then, we introduce two simple performance indices that characterize the motion: (1) the terminal velocity and (2) the acceleration distance. Finally, we present the effect of design parameters on these performance metrics.

In this analysis, we consider the actuation force (i.e., acoustic radiation force) and drag as force applied to the balloon assuming that the buoyancy and the gravity are balanced. Then, We obtain the following equation of motion:

$$
m \dot{v}=F_{\text {arf }}-F_{\text {drag }}
$$

where $m$ is the mass of a balloon, $v$ is its velocity, and a dot over a symbol denotes the time derivetive. Given that the buoyancy equals the gravitational force, the mass can be represented as follows:

$$
m=\frac{4 \pi \rho_{0} R^{3}}{3}
$$

where $\rho_{0}$ is the density of the air. The drag force can be represented as

$$
F_{\text {drag }}=\frac{\pi}{2} \rho_{0} v^{2} R^{2} C_{D}
$$

where $C_{D}$ is the drag coefficient of a smooth sphere. The drag coefficient varies depending on the Reynolds number $R e=\frac{2 \rho_{0} v R}{\mu}$. We assume that the Reynolds number does not exceed the critical Reynolds number, a value at which the boundary layer on the sphere becomes turbulent. This assumption is reasonable considering our

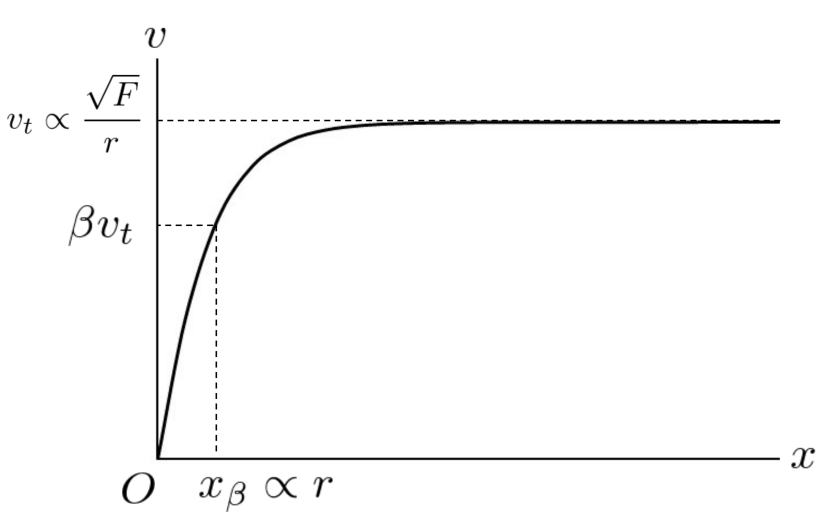

Figure 3: Trajectory in state space.

operation speed (e.g., below $35 \mathrm{~m} / \mathrm{s}$ for a balloon of a $100-\mathrm{mm}$ radius, and $5 \mathrm{~m} / \mathrm{s}$ for a balloon of a $50-\mathrm{mm}$ radius). Given that, we approximate the drag coefficient as follows:

$$
C_{D}=\text { const. }
$$

For example, the drag coefficient of a smooth sphere is 0.4 . Although this approximation ignores the variation of $C_{D}$ at low Reynolds numbers $(R e<1000)$, the approximation error is much smaller than the actuation force (less than $0.05 \mathrm{mN}$ ) and has a negligible effect on the motion. This can be verified by comparing the calculation results of acoustic radiation force with the curve-fit formula for a laminar flow [40].

Substituting Eq. (2) and Eq. (3) into Eq. (1) and solving the equation of motion yields the following solutions:

$$
\begin{aligned}
& v(t)=v_{\mathrm{t}}\left(1-\frac{2}{\mathrm{e}^{2 \mu v_{\mathrm{t}} t}+1}\right) \\
& x(t)=-v_{\mathrm{t}} t+\frac{1}{\mu} \log \left(\frac{\mathrm{e}^{2 \mu v_{\mathrm{t}} t}+1}{2}\right)
\end{aligned}
$$

where $\mu$ is a parameter defined by $\mu=\frac{3 C_{D}}{8 R}$ and $v_{\mathrm{t}}$ is the terminal velocity, at which the drag force and actuation force are balanced. Substituting $\dot{v}=0$ into Eq. (5) yields the terminal velocity as follows:

$$
v_{\mathrm{t}}=\sqrt{\frac{2}{\pi \rho_{0} C_{D}}} \frac{\sqrt{F_{\mathrm{arf}}}}{R} .
$$

Figure 3 shows the trajectory in the state space $(x, v)$.

Here, we introduce two indices that characterize the motion. One is the terminal velocity $v_{\mathrm{t}}$, which we introduced in Eq. (7). This index represents the maximum velocity that the balloon object can achieve after the transient response. If the balloon travels such a long distance that the transient response is negligible, the terminal velocity is the major index that determines the time to travel.

To represent the transient motion, we define the acceleration distance, which is a distance required to reach the velocity $\beta v_{\mathrm{t}}(0<$ $\beta<1$ ). To derive this, we find the acceleration time $\tau_{\beta}$, the time to reach $\beta$ of the terminal velocity, as follows:

$$
\tau_{\beta}=\frac{1}{\mu v_{\mathrm{t}}} \log \left(\frac{1+\beta}{1-\beta}\right) .
$$




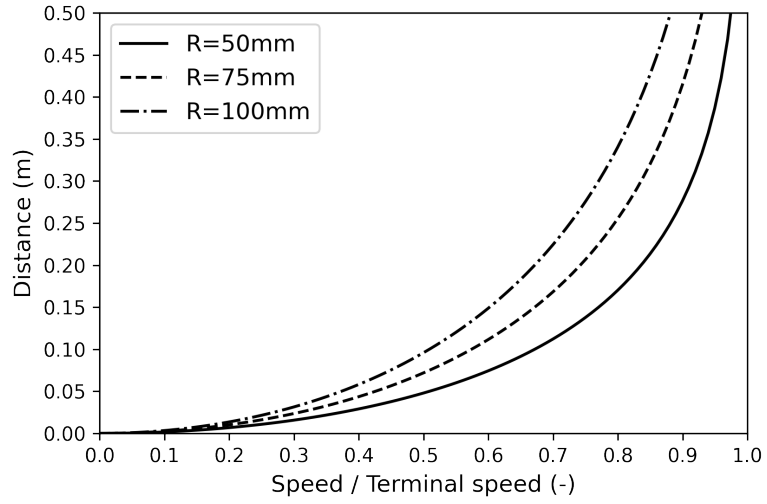

Figure 4: Acceleration distances of balloons of various radii.

Substituting Eq. (8) into Eq. (6) yields the following acceleration distance:

$$
x_{\beta}=\frac{4 R}{3 C_{D}} \log \left(\frac{1}{1-\beta^{2}}\right) .
$$

Note that the acceleration distance depends only on the radius of the balloon. Figure 4 shows the acceleration distances for balloons of various radii. The figure also shows the percentage of the terminal velocity that a balloon can reach within a finite distance. The figure shows that a reachable velocity within a finite distance varies significantly depending on their radii, even if they have the same terminal velocities. For example, within a travelling distance of $100 \mathrm{~mm}$, a balloon of $50-\mathrm{mm}$ radius can reach $67 \%$ of the terminal velocity, whereas a balloon of $100-\mathrm{mm}$ radius can reach $52 \%$. Given that motions around humans are likely to involve travels of such scales, this example illustrates the impact of a transient response on the speed within a finite workspace. The acceleration distance provides a way to evaluate the reachable speed within a provided workspace through a simple calculation.

Now, we discuss how the radius of a balloon $R$ and the actuation force $F_{\text {arf }}$ affect the mobility in terms of the terminal velocity (Eq (7)) and the acceleration distance (Eq. (9)) as indicated in Figure 3. First, the terminal velocity is proportional to the square root of the actuation force and the reciprocal of the radius. Second, the acceleration distance is proportional to the radius of the sphere. From the analysis, we identified that the radius has a significant effect on the reachable speed when the operation involves shortrange travel on the order of $100 \mathrm{~mm}$. This motivates us to use a smaller balloon to achieve a higher speed.

\subsection{Airborne Ultrasound Phased Array}

Ultrasound generates acoustic radiation force when it is reflected. Based on this phenomenon, airborne ultrasound phased arrays (AUPAs) can apply force to a solid object at an arbitrary 3D point without contact by electrically steering an ultrasound beam toward it. We employed AUPAs as noncontact actuators for a midair balloon interface for the following reasons. First, they can apply force to materials frequently used for balloons. Second, their perceivable
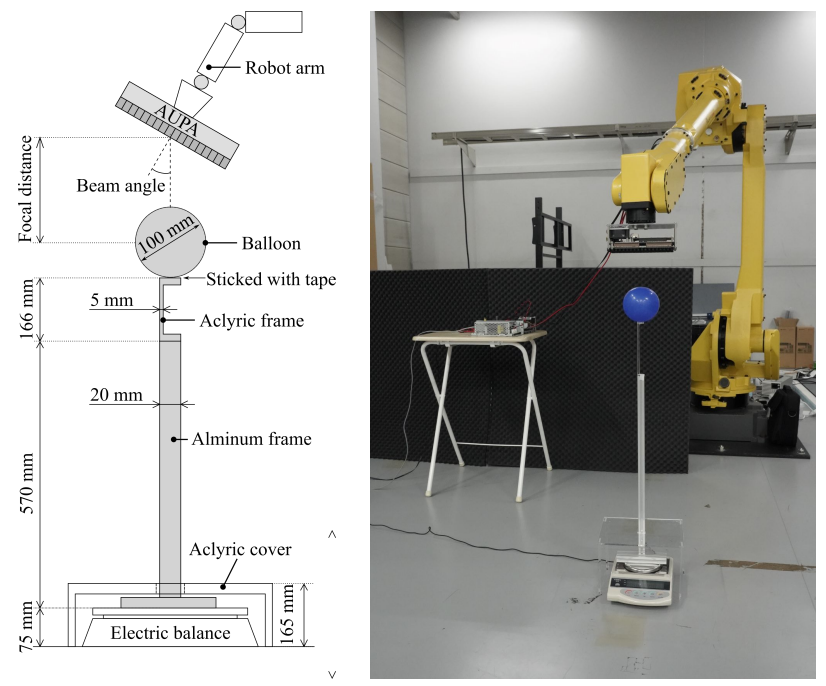

Figure 5: Schematics and photograph of the experimental setup for measurement of the acoustic radiation force on a balloon.

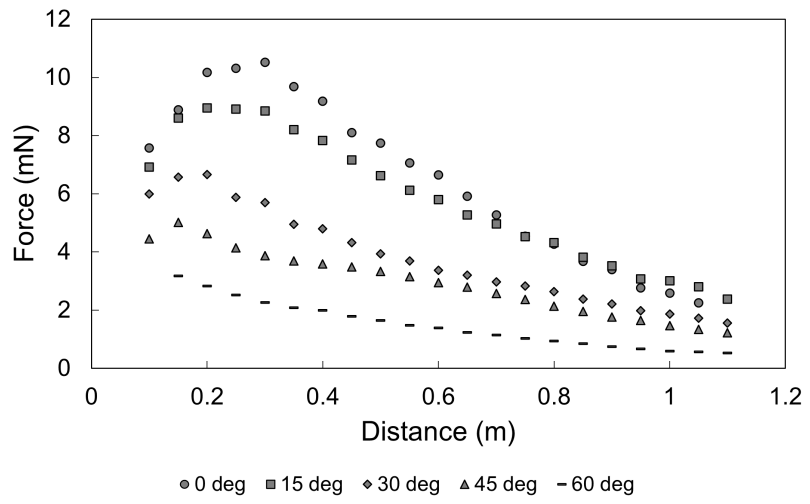

Figure 6: Measured acoustic radiation force on balloon.

artifacts, such as audible sounds or winds, are small. Third, the number of actuators is scalable [18, 36].

We employ AUTD3 [36], a modular AUPA device, because it allows easy synchronization of the driving phases and amplitudes among multiple devices by simply connecting them with Ethernet cables in a daisy chain. Each device has 249 transducers (Nippon Ceramic inc, T4010A1) arranged in a rectangular lattice, resulting in a $182 \mathrm{~mm} \times 141 \mathrm{~mm}$ rectangular aperture. The transducers are driven by $24 \mathrm{Vpp}$ rectangular signals at $40 \mathrm{kHz}$. Further details of the device can be found in [36].

We conducted an experiment to measure the magnitude of acoustic radiation force that a single AUPA device applies to a balloon. Figure 5 shows a schematic figure and a photo of the experimental setup. We used an electric balance (Shinko Denshi, ViBRA AJ2-220) to measure the force. A balloon (Seltenpex, 5' round, dark blue) was mounted on a jig that is designed to alleviate the effect of reflected waves from the ground. We placed an acrylic cover around the 


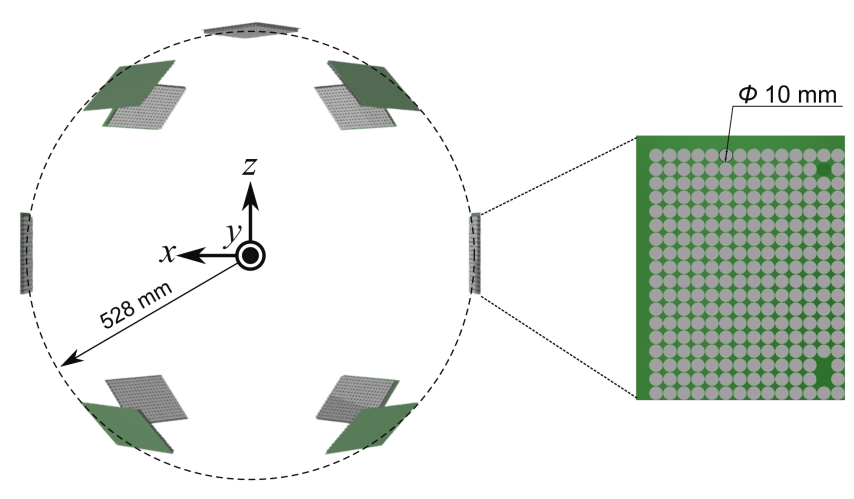

Figure 7: Arrangement of ultrasound transducers.

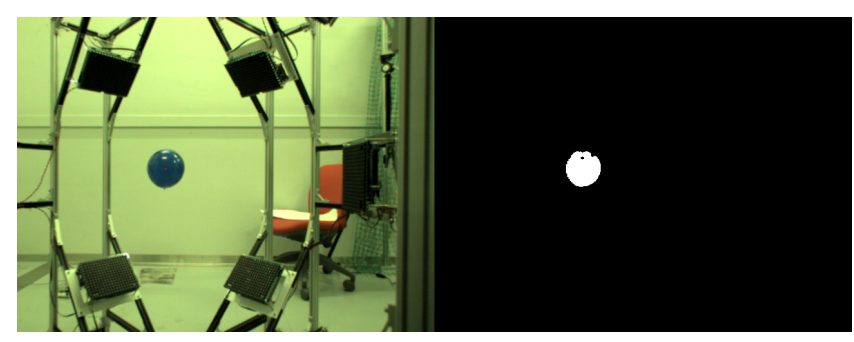

Figure 8: Extraction of balloon from camera image.

electric balance to block the diffracted sound waves. An AUPA device was positioned above the balloon using a robotic arm (FANUC, M710-iC20L). The AUPA device created a focus at the center of the balloon, and we measured the downward force, assuming that the force is repulsive. Figure 6 shows the results. The magnitude depends on the positional relationship between a balloon and an AUPA device.

\subsection{Arrangement of Transducers}

In the arrangement of ultrasound transducers, we should consider the trade-off between the control performance and access to the balloon. To enable three-dimensional control, AUPA devices are arranged such that there are at least three degrees of freedom (DoF) of actuation. While placing AUPA devices in many directions improves the control performance, the devices block the user when she/he tries to see and touch the balloon object inside the workspace. Solving this trade-off is not trivial.

Considering this trade-off, we arrange 11 AUPA devices as shown in Figure 7. They are arranged at a distance of $528 \mathrm{~mm}$ from the center of the workspace. Users can interact with the balloon mainly from the $+y$-direction. To achieve 3-DoF motions without arranging the devices along the y-axis, we directed the eight AUPA devices in the diagonal directions. The other three devices in the $x$ - and $z$-axes provide additional actuation force.

\subsection{Control Algorithm}

We employ a sensor-feedback-control approach [12] to stabilize a balloon in mid-air. This approach changes driving phases and amplitudes of transducers dynamically depending on the measurement of the positional error such that restoring force is applied to the controlled object.

First, the algorithm calculates restoring force using a PositionalIntegral-Differential (PID) controller. It calculates the force to follow a target trajectry $\boldsymbol{r}_{\text {tgt }}(t)$ based on the positional error $\Delta \boldsymbol{r}=\boldsymbol{r}-$ $\boldsymbol{r}_{\text {tgt }}(t)$, the velocity error $\Delta v=\dot{\boldsymbol{r}}-\dot{\boldsymbol{r}}_{\text {tgt }}(t)$ and the second time derivative of the target position $\boldsymbol{a}_{\mathrm{tgt}}=\ddot{\boldsymbol{r}}_{\mathrm{tgt}}(t)$ as follows:

$$
\begin{aligned}
\boldsymbol{a} & =\boldsymbol{a}_{\mathrm{tgt}}-k_{\mathrm{p}} \Delta \boldsymbol{r}-k_{\mathrm{d}} \Delta v-k_{\mathrm{I}} \int \Delta \boldsymbol{r} d t \\
\boldsymbol{F}_{\mathrm{tgt}} & =m \boldsymbol{a}
\end{aligned}
$$

where $k_{\mathrm{p}}, k_{\mathrm{d}}$, and $k_{\mathrm{I}}$ are user-defined parameters. Throughout this paper, we set these parameters to $32 s^{-2}, 20 s^{-1}$, and $0.05 s^{-3}$ respectively.

Next, the algorithm calculates driving phases and amplitudes that generate restoring force calculated by the PID controller. The previous algorithm [12] sets the driving amplitudes such that the linear summation of force provided by each single AUPA device equals the desired force, and drives all the transducers simultaneously. This process implicitly assumes that there is no interference among the ultrasound beams. When ultrasound beams impinge on points that are closer than the beam width, interference occurs and produces unmodelled errors that degrade the control performance. Because interference is more likely to occur when we control a small balloon with denser arranged AUPA devices, the previous algorithm is unsuitable for our study. To eliminate interference, we introduce time-sliced driving of AUPA devices. The time-sliced driving technique divides the time domain into time slices of a fixed length. In each time slice, only one device is driven. Although the balloon can propel only in 11 directions within each time slice, fast switching of the AUPA devices produces time-averaged force in an arbitrary direction, allowing movements along curved trajectories as demonstrated later in Chapter 5. Throughout the paper, the length of a time slice was set to $10 \mathrm{~ms}$.

Our algorithm creates focus on the balloon by setting the phases as follows:

$$
\phi_{l}=k\left|\boldsymbol{r}-\boldsymbol{r}_{l}\right|
$$

where $k$ is the wave number, and $\boldsymbol{r}$ and $\boldsymbol{r}_{l}$ are the positions of the balloon and the $l$-th transducer, respectively. Focusing beam produces acoustic radiation force in a repulsive direction.

Our algorithm determines the driving power of each AUPA device, which is proportional to the square of the driving amplitude, through the following steps. First, it optimizes the time-averaged power of the AUPA devices $\overline{\boldsymbol{u}}=\left[\bar{u}_{1} \bar{u}_{2} \ldots \bar{u}_{N}\right]^{T}$ such that the timeaveraged net force exerted by all the AUPA devices equals the desired force as follows:

$$
\begin{gathered}
\overline{\boldsymbol{u}}^{*}=\underset{\overline{\boldsymbol{u}}}{\arg \min }\left|\boldsymbol{F}_{\mathrm{tgt}}-\sum_{\mathrm{i}} \bar{u}_{i} \boldsymbol{F}_{i}(\boldsymbol{r})\right|^{2} \\
0 \leq \bar{u}_{i}
\end{gathered}
$$

where $\boldsymbol{F}_{\text {tgt }}$ is force to apply and $\boldsymbol{F}_{i}$ is the acoustic radiation force applied by the $i$-th AUPA device when it is driven at a unit power. Because the acoustic radiation force is a function of the relative position between the balloon and the AUPA, the value of $\boldsymbol{F}_{i}$ is estimated by the current position of the balloon. To achieve fast estimation, the algorithm estimates the value by linear interpolation 

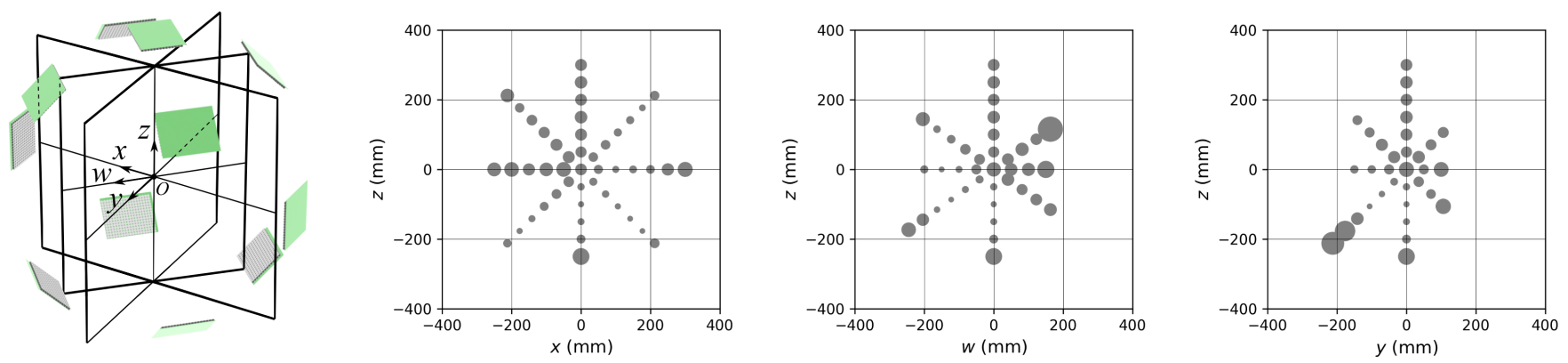

Figure 9: Accuracy and workspace provided by prototype. Size of circle indicate average error. Positions and sizes of circles are described in a consistent scale. (a) Definitions of planes (a) and accuracy in the $x y$-plane (b), the $w z$-plane (c), and the $y z$-plane.

of the measured values in our experiment (Figure 6). Then, the time slices are assigned to AUPA devices to be driven. Multiplying the time-averaged power by the number of driven AUPA devices yields the actual driving power as follows:

$$
u_{i}^{*}=n \bar{u}_{i}^{*}
$$

Throughout the paper, the update rate of the driving phase and amplitudes was set to $100 \mathrm{~Hz}$.

\subsection{Tracking a Balloon}

We use two high-speed cameras (MQ003CG-CQ, Ximea GmbH) to track the balloon. Their camera parameters and the positional and rotational relationship between the two cameras are estimated using circle grid patterns. Their poses relative to the AUPA setup are estimated by measuring and least-square fitting [5] of the positions of 20 markers attached to the setup. The system extracts the balloon from the camera image by backprojecting the hue histogram of its reference image and then binarizing through a thresholding [37]. Triangulating the centers of mass of the processed images yields the 3D position of the balloon. Calibration and image processing described above are implemented using the OpenCV library ${ }^{1}$. The resulting tracking rate was $177 \mathrm{fps}$.

\section{EVALUATION OF MOBILITY}

In this chapter, we describe the evaluation of mobility provided by our prototype system. As a balloon object, we used a balloon of a 100mm diameter (Seltenpex, 5' round, dark blue) in the experiments. The balloon was inflated with helium gas. We attached masking tape such that the buoyancy and gravity were balanced.

We conducted an experiment to evaluate the accuracy of the proposed control method. The balloon was initially positioned at the origin. It was then moved by $50 \mathrm{~mm}$ in eight directions in the $x z^{-}, y z^{-}$, and $w z$-planes every $20 \mathrm{~s}$ (Figure 9). At each position, we waited for $5 \mathrm{~s}$ for the balloon to complete the transition. We then recorded the position for the remaining $15 \mathrm{~s}$ to measure the error of the position. The series was finished when the stabilization failed. The experiment was conducted in a room with limited airflow. The room temperature was $24^{\circ} \mathrm{C}$.

Figure 9 shows the average error at each target position. The farthest point plotted in the graph indicates the size of the workspace

\footnotetext{
${ }^{1}$ https://opencv.org/
}

of the prototype system. The center and radius of each circle represent the target position and average error respectively. The radius and the position are drawn at a consistent scale. From the figures, it can be seen that the reachable distance from the origin depends on the direction. In the $x z$-plane, the balloon was stabilized at a distance of more than $250 \mathrm{~mm}$ in every direction. The position error was less than $22 \mathrm{~mm}$. The reachable distance in the $y$-direction was the smallest among the moving directions tested, resulting in $100-150 \mathrm{~mm}$ from the origin. The maximum average error in the $y$-direction was $19.1 \mathrm{~mm}$.

Next, we conducted an experiment to test the speed. A balloon of a $10-\mathrm{cm}$ diameter was moved $500 \mathrm{~mm}$ in the $+x-,+z-$, and $-z$ directions. Each maneuver was repeated 7 times. For the horizontal maneuvers, the average translation time was $1.36 \mathrm{~s}$, recording an average peak velocity of $890 \mathrm{~mm} / \mathrm{s}$. For the vertical maneuvers, the average translation times were $1.54 \mathrm{~s}$ in the $+z$-direction and $1.44 \mathrm{~s}$ in the $-z$-direction, recording average peak velocities of $671 \mathrm{~mm} / \mathrm{s}$ and $798 \mathrm{~mm} / \mathrm{s}$ respectively.

Finally, we conducted experiments to test the ability to control a balloon along curved trajectories. We tested three curved trajectories in the $x z$-plane: a circular trajectory, a $\infty$-shaped trajectory, and a heart-shaped trajectory. Their periods were set to $3 \mathrm{~s}, 5 \mathrm{~s}$, and $8 \mathrm{~s}$ respectively. Figure 10 shows the recorded trajectories. These figures indicate that the prototype system can control the balloon along a circular trajectory, inferring its applicability to curved trajectories.

\section{INTERACTION}

\subsection{Sight-based Interaction}

We implemented a sight-based interaction, in which a balloon object is situated within the user's view rather than using the global coordinates. The balloon is controlled such that the user sees the balloon at a constant direction when the pose of the user's head is changed.

We used a commercial tracking sensor (Natural Point, Track IR and TrackClip) to measure the pose of the head. The pose of the head is fed into the PID controller described in Section 4.4. Figure 12 shows how the user sees a balloon object along with the angles between the face direction and the direction to the balloon, as well as the angular speed of the user's head when the user rotates his/her head vertically and horizontally $300 \mathrm{~mm}$ away from the 

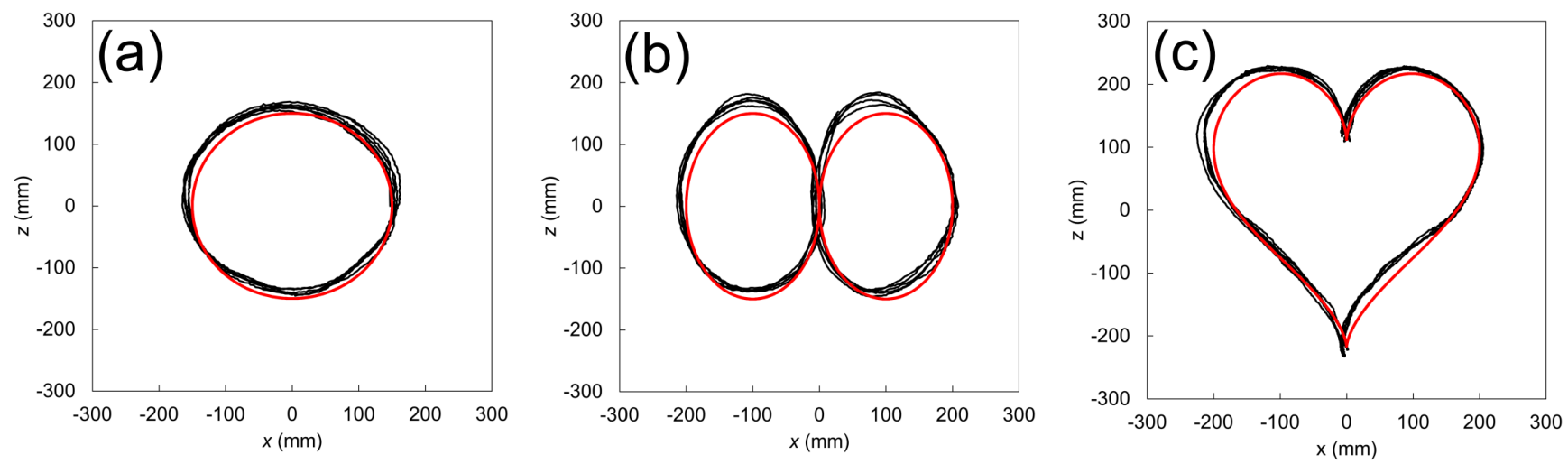

Figure 10: Curved trajectories. Black and red lines indicate recorded and target trajectories respectively. Each figure contains 5 loops. (a) Circular trajectory of a 3-s period. (b) $\infty$-shaped trajectory of a 5-s period. (c) Heart-shaped trajecotry of a 8-s period.

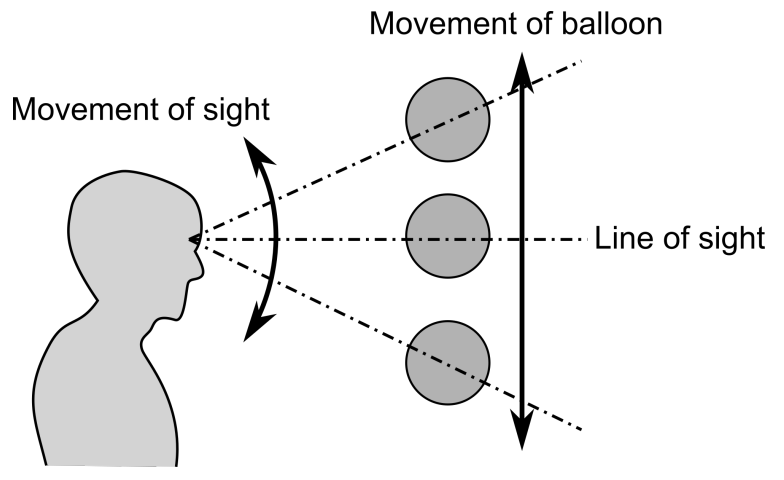

Figure 11: Sight-based interaction.

origin. The figure shows that the balloon is positioned in the same place inside the view, although a delay of approximately $1 \mathrm{~s}$ occur (see the supplementary video).

We note that the motion in this experiment was slower than that observed in the mobility tests (Chapter 5) Actually, the peak speeds were $306 \mathrm{~mm} / \mathrm{s}$ in the horizontal motion and $189 \mathrm{~mm} / \mathrm{s}$ in the vertical motion. The main cause of this difference in speed is the PID control algorithm. In PID control algorithm, actuation force has a term that is proportional to the distance to the destination (10). In the mobility tests, the distance to the destination was set to a large value (i.e. $500 \mathrm{~mm} / \mathrm{s}$ ) at the beginning. This caused the PID controller to apply large actuation force to the balloon. In the experiments of a sight-based interaction, the distance to the destination was below $200 \mathrm{~mm}$ in the horizontal motion and $120 \mathrm{~mm}$ in the vertical motion because the destination gradually changed as the user's face moved keeping. This resulted in small actuation force that is well below the maximum force provided by the prototype. This implies that a control algorithm that fully exploits the capability of the actuators would allow the balloon to follow the user's motion more quickly.

\subsection{Touch-based Interaction}

6.2.1 Touch-based Input. We implemented touch-based interactions that resemble our object manipulation in our daily lives. A
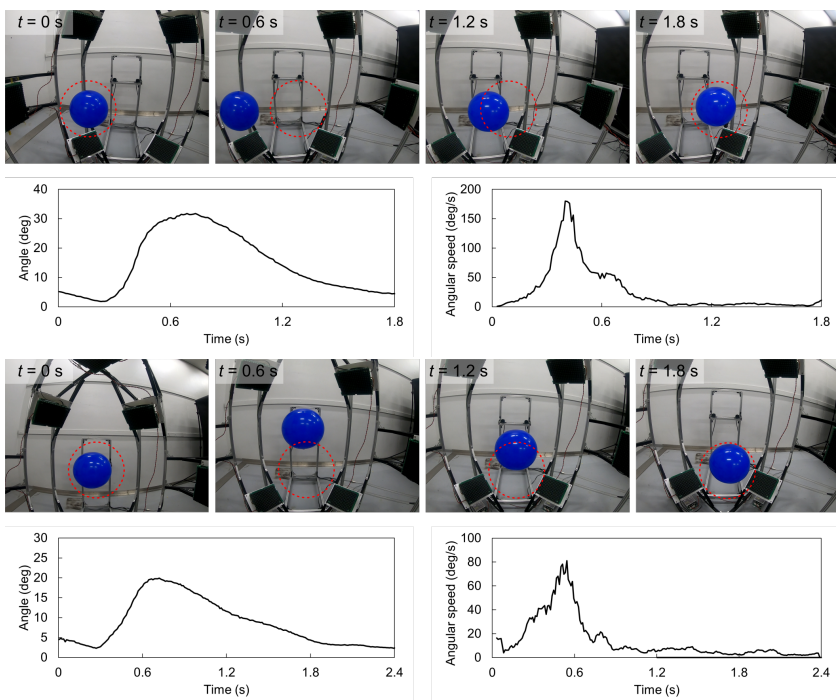

Figure 12: First person view captured with head-mounted camera during sight-based interaction (GoPro, HERO 07) when user rotates head horizontally (Top) and vertically (Bottom). Graphs below photos show angle between direction of face and direction toward balloon object (Left) and angular speed of user's haed (Right).

user can touch, grasp, and translate the balloon to an arbitrary 3D point within the workspace directly with the user's hands. Furthermore, to facilitate a command input in mid-air, we implemented a "click" gesture by which the user can invoke an event through the movement of the finger on the surface of the balloon. We call this input technique "click" because it is analogous to the click of a 2D mouse, although the movement of the finger is inversed. Through a click motion, the balloon acts as the reference surface of the motion, which tells the user where the motion should be made. This is contrary to midair gestures in that the motion tends to be large because of the ambiguity of the reference position. 
To detect the user's motion, we implemented a sensing system that discriminates the following four contact states: free, touch, grasp (finger down), and grasp (finger up) (Figure 13). The manipulation of a balloon causes the transition from a free state to a grasp state initially, and then the inverse transition occurs at a different position at the end. The system interprets these transitions as a manipulation and starts controlling the balloon at a new position at which the balloon is released. The system detects a clicking motion when the transitions between the finger-down and finger-up states occur within a predefined period. This period is set to $0.5 \mathrm{~s}$ for our prototype.

We used stereo cameras and a depth sensor (Intel, RealSense D435) to detect the user's actions. The point cloud acquired by the depth sensor is processed using a median filter, a range thresholding, a spatial filter, and a temporal filter provided by RealSense. Then, the point cloud was down sampled using a voxel grid filter with a 5-mm leaf size. A Touch is detected if the number of points inside a collider registered just outside the balloon is counted based on the distance from its center tracked by the stereo cameras. The user's hold is detected if the touch is detected in 7 consecutive frames. The system detects the user's click motion based on the distance between the balloon and the edge of the nearest cluster in the $z$ direction. If the difference exceeds a specified threshold, the user's click is detected. These processes were implemented using Point Cloud Libraries [33], unless otherwise specified. In our prototype, we set the touch and click ditection thresholds to $3 \mathrm{~cm}$ and $5 \mathrm{~cm}$ respectively.

6.2.2 Vibrotactile Feedback. An ability to provide two or more types of haptic sensations is useful for input devices. For example, when a user clicks a mouse button, the user finds the neutral position by feeling contact and confirms the completion of the action by the distinct sensation of click. Using different haptic sensations for indicating a neutral position and an action completion facilitates intuitive and quick input actions.

To present multiple types of haptic sensations, we propose a vibrotactile feedback method for midair balloon interfaces. Our method provides vibrotactile sensation by vibrating the balloon in a hand with amplitude-modulated ultrasound (Figure 15), which applies sinusoidally varying force to the balloon. This method is based on our finding that ultrasound can produce perceivable vibrations of a balloon in hand when the ultrasound is amplitude-modulated at a frequency that is sensitive to cutaneous mechanoreceptors of humans. (approximately $200 \mathrm{~Hz}$ [21]). The perceived strength can be varied by changing the driving power of the ultrasound transducers. This method can be utilized to notify events that occur when the balloon is held by users. For example, it can be utilized to produce 'click sensation' for the click gesture described in section 6.2.1. In this paper, we set the modulation frequency to $200 \mathrm{~Hz}$.

\section{EVALUATION OF VIBROTACTILE \\ FEEDBACK}

We conducted a psychophysical experiment to evaluate the perceived strength of the proposed vibrotactile feedback method described in Section 6.2.2 (Figure 15). In this experiment, we measured absolute detection threshold and just noticeable difference. The absolute detection threshold is a minimum power to produce the

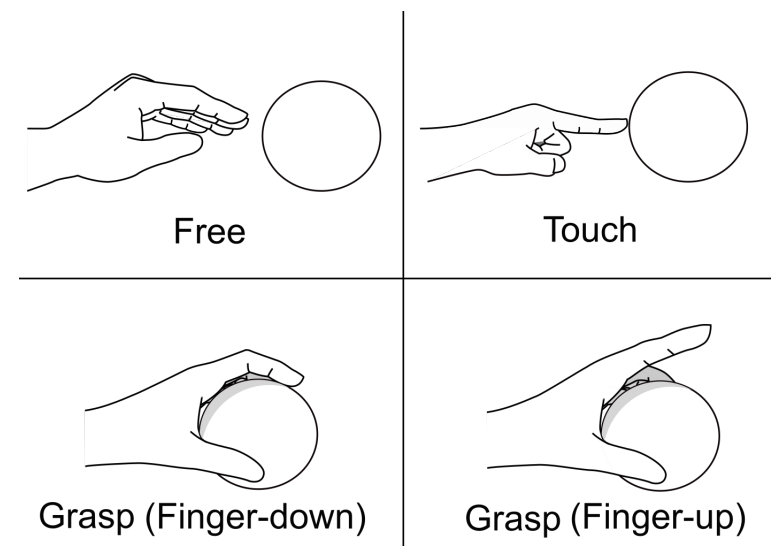

Figure 13: Hand states.

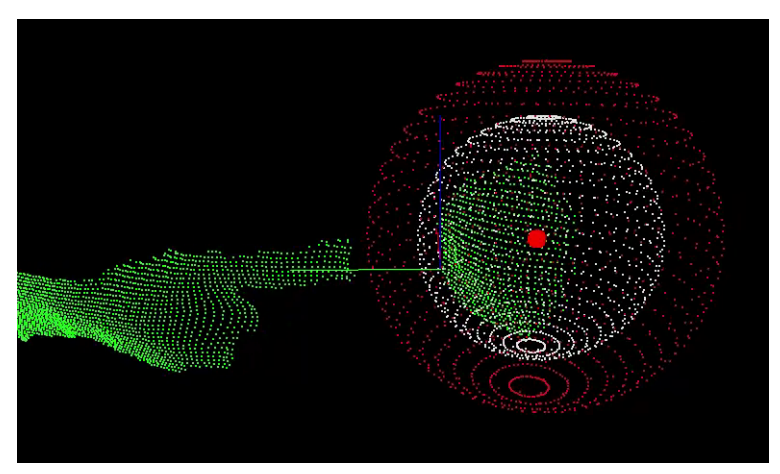

Figure 14: Point cloud obtained by depth sensor. Green points are processed points obtained by depth sensor. Red dot is estimated center of balloon obtained by stereo RGB cameras. White and red sphere are colliders used to detect contact between finger and balloon.

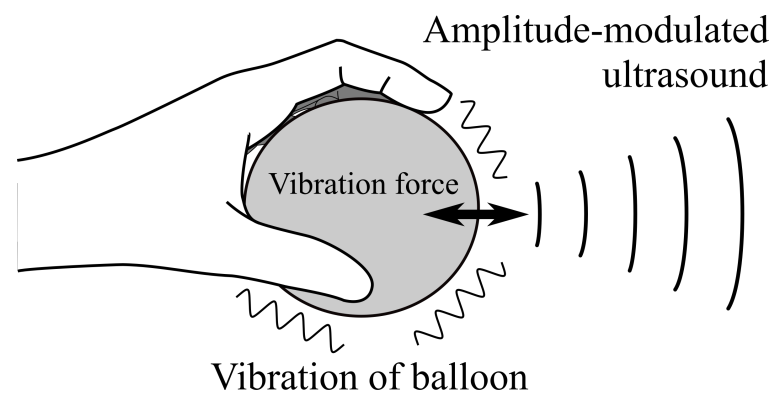

Figure 15: Principle of vibrotactile feedback.

perceivable vibration of the balloon in the user's hand. At this driving power, the prototype produces vibrations that are barely perceivable. This value is the baseline of the perception of the vibrotactile stimulation. The just noticeable difference is the minimum difference of power required to produce perceptually different strengths of the vibrotactile sensation. From these values and the 


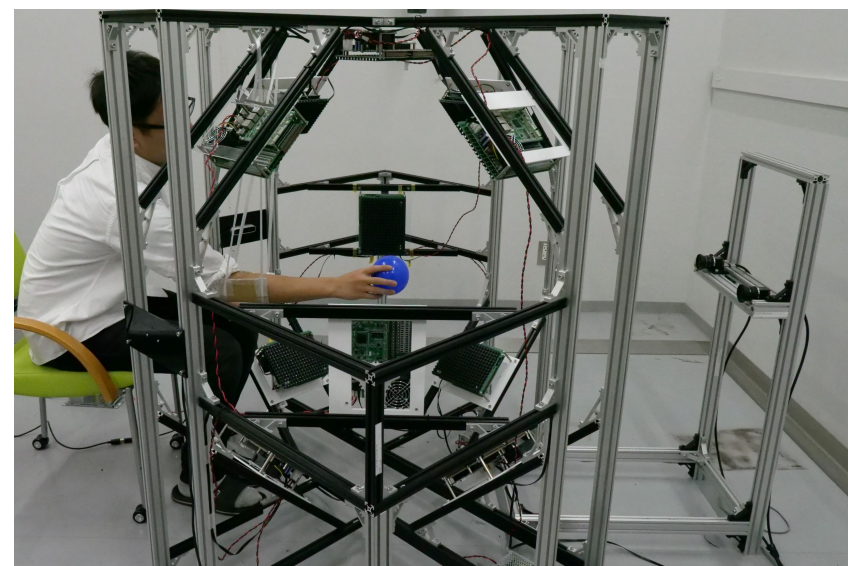

Figure 16: Experimental setup for measuring absolute detection threshold and just noticeable difference of vibrotactile feedback.

maximum available power, we can estimate the range of perceived strength that the prototype can provide.

The perceived strength may be affected by the number of contact fingers because the fingers act as points at which the user feels the vibration as well as points at which the physical vibration of the balloon is constrained. In this experiment, we asked participants to hold a balloon by pinching it with two fingers or by grasping it with five fingers and measured the perceived strength for both cases. We chose the two conditions because a sphere is typically held in these ways.

All procedures were in accordance with the Declaration of Helsinki, and participants provided written informed consent prior to the study.

\subsection{Experimental Setup}

Figure 16 shows the experimental setup. The participant sat in the $+y$-direction of the prototype setup and held a balloon (Seltenpex, 5 ' round, dark blue) of a $10-\mathrm{cm}$ diameter filled with helium gas with his/her dominant hand. The participant positioned the balloon approximately at the origin of the workspace and rested a forearm on an armrest hanging on the prototype setup. The participant wore headphones playing pink noise to mask out audible noise from the AUPA devices. The experiments were conducted in a quiet, bright room with a limited airflow. The room temperature was between $22^{\circ} \mathrm{C}$ and $24^{\circ} \mathrm{C}$.

When vibrotactile stimulation was provided, all the AUPA devices creates a focus at the center of the balloon. We used a tracking system described in Section 4.5 to measure the center of the balloon. All transducers were driven at the same amplitudes. The ultrasound was amplitude-modulated at $200 \mathrm{~Hz}$.

\subsection{Procedure}

7.2.1 Measurement of Absolute Detection Threshold. In the measurement of the absolute detection threshold, we employed the method of limits with three ascending and descending series. In the ascending series, the initial power was sufficiently small such

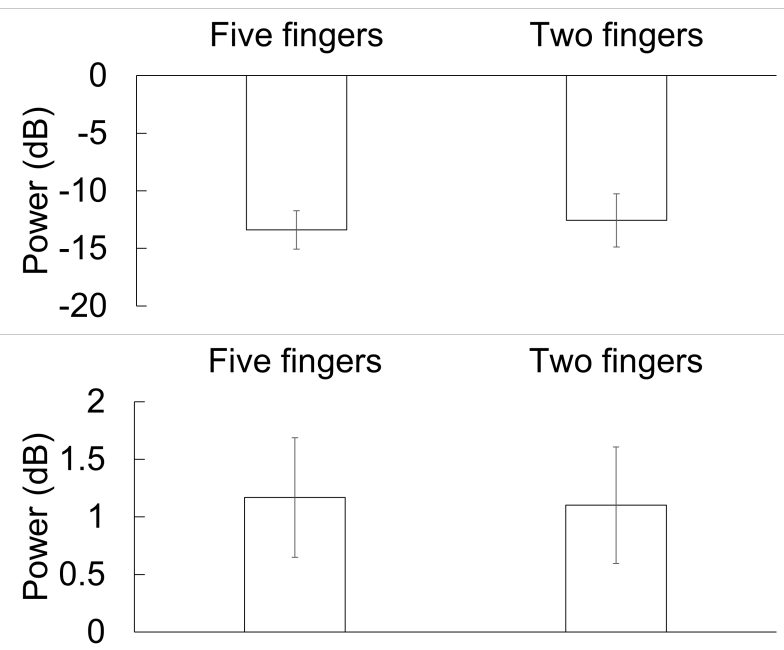

Figure 17: Absolute detection threshold (Top) and just noticeable difference (Bottom) of vibrotactile feedback.

that the stimulus was not perceived. Conversely, in the descending series, the initial power was sufficiently large such that the stimulus was perceived. The initial power was randomized in steps to reduce the expectation bias for each series. The step size was set to $1 \mathrm{~dB}$.

A stimulation was presented for $1 \mathrm{~s}$. The beginning and end of the stimulation were informed by a beep sound of $500 \mathrm{~ms}$ in length. The participants were asked to answer whether they detected the stimulus verbally after the presentation. After the participant answered, the experimenter proceeded with the experiment. Each series was completed when the answer changed. Before the experiment, one ascending series and one descending series were applied to ensure that the participants understood the required task and stimulus to detect.

A total of 15 participants (2 females and 13 males, aged 23-36, mean of 26.2) participated in the experiment. All participants were right-handed.

7.2.2 Measurement of Just Noticeable Difference. To measure the just noticeable difference, we employed a three-interval onealternative forced-choice procedure [23]. During each trial, two reference signals and one target signal were presented in a randomized order. Before and after the stimulation, beep sounds were provided to notify the participant about the beginning of the stimulation. Each stimulation was provided for $600 \mathrm{~ms}$. The signals are separated by intervals of $200 \mathrm{~ms}$. A participant was asked to answer the target signal verbally. The initial power of the target signal was set to a sufficiently large value such that the signal could be easily discriminated. Every time she/he answered correctly three times successively, the power of the target stimulus was decreased by a predefined step. Once the participant answered incorrectly, the power of the target stimulus was increased by a predefined step. The step size was initially set to $0.4 \mathrm{~dB}$ for a fast search of the just noticeable difference value and was reduced to $0.1 \mathrm{~dB}$ after the first reversal. An experimental run was completed when three reversals occurred at the small step size. The average of the value at the reversals resulted in an estimate of the just noticeable difference. 
A total of 14 participants ( 2 females and 12 males, aged 23-29, mean of 25.0) participated in the experiment. All participants were right-handed.

\subsection{Results}

In both holding conditions, the stimulation was clearly perceived by all participants. Figure 17 shows the results of the absolute detection thresholds for the two holding conditions. Each bar indicates the average threshold in $\mathrm{dB}$ units. $0 \mathrm{~dB}$ indicates the maximum power provided by the prototype setup. In both conditions, the values of absolute detection tholeshold were much smaller than the maximum power (a mean of $-13.4 \mathrm{~dB}$, and an SD of $1.68 \mathrm{~dB}$ for the five-fingers condition and a mean of $-12.6 \mathrm{~dB}$, and an SD of 2.3 $\mathrm{dB}$ for the two-fingers condition). The estimated just noticeable difference was $1.18 \mathrm{~dB}$ (SD of $0.52 \mathrm{~dB}$ ) for the five-fingers condition and $1.22 \mathrm{~dB}(\mathrm{SD}$ of $0.51 \mathrm{~dB}$ ) for the two-fingers condition. These results indicate that the prototype can vary the strength of the vibrotactile stimuli within a range of approximately 11 distinct steps when a user grasps the balloon with five fingers and approximately 10 distinct steps when a user pinches with two fingers.

\section{APPLICATIONS}

\subsection{Authoring 3D Motions}

A midair balloon interface can be used as a '3D mouse', with which a user can specify a 3D path by physically moving a balloon and issue a command by 'clicking' it.

For example, imagine a situation where an artist designs a performance that uses a drone equipped with LEDs (Figure 18 (a)). She needs to plan the trajectory of the drone and the timing of the blinking of the LEDs while being careful to stay inside the exhibition space. While prototyping motions with read drone is effective in design process, prototyping such a complex sequence is a difficult task for non-expert pilots. A balloon interface can be utilized as a '3D mouse' for authoring the motion. The artist can specify a desired 3D path by holding the balloon and moving it along the path. She can specify the timing of the blinking of the LEDs by "clicking". Vibrotactile feedback can be used to indicate that the drone is approaching the edge of the exhibition space.

While we described the example of controlling an aerial vehicle here, a midair balloon interface can be applied to 3D motion design of other things such as a virtual character in virtual reality and augmented reality.

\subsection{Telepresence Avatar and Robotic Character for Story Telling}

A midair balloon interface can be used to present an avatar of a remote user in telepresence applications or a character in storytelling applications by projecting its face (Figure 18 (b)). Spatial movement and interaction distance can convey nonverbal cues that are useful in real-world communications. For example, a balloon-projected character can agree or disagree by moving the body vertically or horizontally and express surprise by 'jumping' motion (quickly ascending and descending). It can also express intimacy by safely moving to the proximity of a person. It produces no audible sound that disrupts conversations.

\subsection{Assembly, Repair, and Training}

Augmented reality has demonstrated its advantage to present technical information in assembly, repair, and training applications. Typically, head-mounted displays and personal digital assistants are used as displays to present information. In these scenarios, it is desirable that displays are lightweight to minimize workers' fatigue. A midair balloon interface can be used as a device-free projectionbased display. Figure 18 (c) illustrates a midair balloon interface that displays instructions in a repair scenario. In this scenario, the midair balloon interface is positioned itself in front of the user's face and within easy reach, allowing touch-based interactions and preventing AUPA devices from being occluded by the workpiece. When instructions are presented, the balloon first comes inside the field of view. Then, the balloon guides the worker to the part of interest by moving toward it. After that, the balloon begins the instructions, allowing the user to see the instructions and the part of interest simultaneously. When the worker starts working, the worker can move the balloon to a convenient place directly with his/her hand to clear the workplace. After he/she finished the task, he/she can confirm the completion of the task by clicking on the balloon. Then, the balloon interface returns to the workspace and begins the next instructions. Because it has no supporting structures, it does not occupy or occlude the workplace more than required to present information.

\subsection{Augmented Sports}

Balloons have long been used in ball games, particularly for children, partly because they are soft and safe. A midair balloon interface provides an opportunity to create ball games of artificial dynamics. Figure 18 (d) shows the use of a midair balloon interface for playing catch under augmented dynamics, where a ball released downward falls upward. As shown in this figure, even such unnatural dynamics can be programmed, providing new experiences to the players. The safety and form factors allow players to grasp and release a ball naturally. Such experiences are basic but difficult to provide with previous midair interfaces. A player can also inflate a balloon larger than a hand and use it for volleyball-like games. The flexibility of the form factor is another benefit in the design of augmented ball games.

\section{LIMITATIONS AND FUTURE WORKS}

\subsection{Mobility}

In this study, we proposed an algorithm to propel a balloon using a single AUPA device at a time, eliminating the effect of interference between ultrasound beams. Although we believe this is a good starting point for the phase computing algorithm, there is still room to improve the propulsive force by optimizing the driving of all transducers, leveraging the interference between the beams. To achieve this, we need a method to compute such driving that optimizes the resultant force of a scattered wave fast enough to achieve a 3D stabilization through sensor feedback. (Note that online methods optimize not the resulting force but rather the pressure distribution of a sound field without scattering.) 
(a)
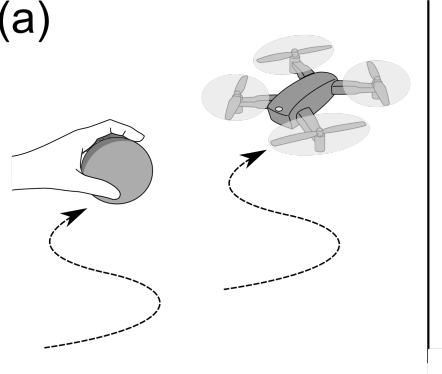

(b)

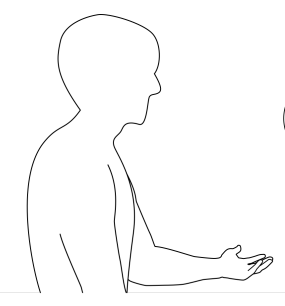

(c)

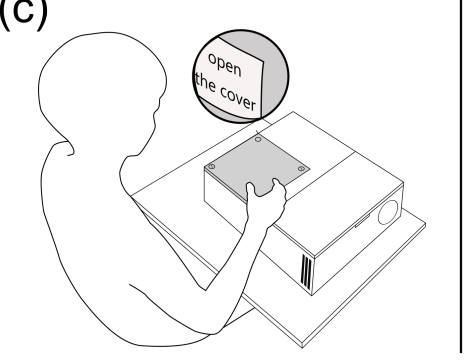

(d)

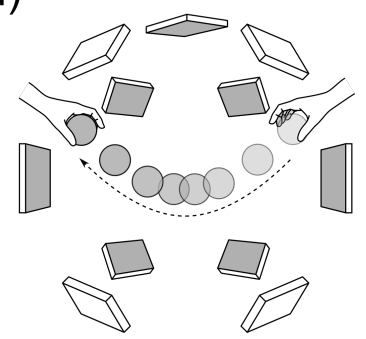

Figure 18: Applications of midair balloon interface: (a) Designing 3D trajectory of aerial vehicle, (b) Projecting a character for tele-conferencing or story-telling, (c) Projecting instrucions for assembly, repair, and maintance tasks, and (d) augmented sports.

\subsection{Manipulating Objects Beyond Balloons}

In this paper, we use a helium-filled balloon whose buoyancy and gravity are balanced. To extend the choice of a midair object, the gravity should be counteracted by AUPAs. Currently, AUPA can provide the actuation force up to $10 \mathrm{mN}$. It can be improved by optimizing driving phases, using more powerful transducers, and increasing the number of transducers.

\subsection{Scalability}

Increasing the distance between an AUPA device results in a smaller actuation force owing to an attenuation. Empowering the power of the transducers and increasing the number of transducers can compensate for this effect. Increasing the workspace to an extent that it surrounds the body increases the chance of entering the ultrasound to human ears. In this case, we should evaluate the safety of the ears and consider measures to prevent strong ultrasound from entering the ears. Safety measures include wearing earphones and controlling transducers such that the sound level around the humans is below the acceptable levels.

\subsection{Easy Manipulation of a Balloon}

Although users can manipulate a balloon easily in almost every case, we found that they sometimes hit the balloon and pop it outside the workspace unintentionally, failing to grasp it. This can be prevented by enlarging the workspace to catch the popped balloon or by enforcing the actuation force by increasing the number of transducers or an algorithm to compute the driving phases and amplitudes.

\section{CONCLUSION}

In this paper, we introduced midair balloon interfaces, a new approach to providing a midair object that is safe and has a compelling speed. This approach is based on the noncontact actuation of a balloon using airborne ultrasound transducers installed in the environment. We analyzed the dynamics of the midair balloon interface and clarified the relationship between the design parameters and the reachable speed. We presented a control algorithm that can cover a wider range of balloon sizes than previously available, allowing the use of a small balloon. We also presented sight-/touch-based interactions and a vibrotactile feedback method. We implemented these functions on a prototype with multiple phased arrays and high-speed cameras. Our user study showed the prototype system can provide vibrotactile sensations at several distinct steps of strength. Finally, we discussed the applications that benefit from a midair balloon interface.

\section{ACKNOWLEDGMENTS}

This work was supported in part by KAKENHI Grant Number JP21K17782 and JST, CREST Grant Number JPMJCR18A2.

\section{REFERENCES}

[1] Parastoo Abtahi, Benoit Landry, Jackie (Junrui) Yang, Marco Pavone, Sean Follmer, and James A. Landay. 2019. Beyond The Force: Using Quadcopters to Appropriate Objects and the Environment for Haptics in Virtual Reality. In Proceedings of the 2019 CHI Conference on Human Factors in Computing Systems (Glasgow, Scotland Uk) (CHI '19). Association for Computing Machinery, New York, NY, USA, 1-13. https://doi.org/10.1145/3290605.3300589

[2] Parastoo Abtahi, David Y. Zhao, Jane L. E., and James A. Landay. 2017. Drone Near Me: Exploring Touch-Based Human-Drone Interaction. Proc. ACM Interact. Mob. Wearable Ubiquitous Technol. 1, 3, Article 34 (Sept. 2017), 8 pages. https: //doi.org/10.1145/3130899

[3] Tobias Alrøe, Jonas Grann, Erik Grönvall, Marianne Graves Petersen, and Jesper L. Rasmussen. 2012. Aerial Tunes: Exploring Interaction Qualities of MidAir Displays. In Proceedings of the 7th Nordic Conference on Human-Computer Interaction: Making Sense Through Design (Copenhagen, Denmark) (NordiCHI '12). Association for Computing Machinery, New York, NY, USA, 514-523. https://doi.org/10.1145/2399016.2399095

[4] Marco A. B. Andrade, Asier Marzo, and Julio C. Adamowski. 2020. Acoustic levitation in mid-air: Recent advances, challenges, and future perspectives. Applied Physics Letters 116, 25 (2020), 250501. https://doi.org/10.1063/5.0012660 arXiv:https://doi.org/10.1063/5.0012660

[5] K S Arun, T S Huang, and S D Blostein. 1987. Least-Squares Fitting of Two 3-D Point Sets. IEEE Transactions on Pattern Analysis and Machine Intelligence PAMI-9, 5 (1987), 698-700. https://doi.org/10.1109/TPAMI.1987.4767965

[6] Sean Braley, Calvin Rubens, Timothy Merritt, and Roel Vertegaal. 2018. GridDrones: A Self-Levitating Physical Voxel Lattice for Interactive 3D Surface Deformations. In Proceedings of the 31st Annual ACM Symposium on User Interface Software and Technology (Berlin, Germany) (UIST '18). Association for Computing Machinery, New York, NY, USA, 87-98. https://doi.org/10.1145/3242587.3242658

[7] Tom Carter, Sue Ann Seah, Benjamin Long, Bruce Drinkwater, and Sriram Subramanian. 2013. UltraHaptics : Multi-Point Mid-Air Haptic Feedback for Touch Surfaces. Proc. UIST 2013 (2013), 505-514. https://doi.org/10.1145/2501988.2502018

[8] Sara Eriksson, Åsa Unander-Scharin, Vincent Trichon, Carl Unander-Scharin, Hedvig Kjellström, and Kristina Höök. 2019. Dancing With Drones: Crafting Novel Artistic Expressions Through Intercorporeality. In Proceedings of the 2019 CHI Conference on Human Factors in Computing Systems (Glasgow, Scotland 
Uk) (CHI '19). Association for Computing Machinery, New York, NY, USA, 1-12. https://doi.org/10.1145/3290605.3300847

[9] Euan Freeman, Asier Marzo, Praxitelis B. Kourtelos, Julie R. Williamson, and Stephen Brewster. 2019. Enhancing Physical Objects with Actuated Levitating Particles. In Proceedings of the 8th ACM International Symposium on Pervasive Displays (Palermo, Italy) (PerDis '19). Association for Computing Machinery, New York, NY, USA, Article 2, 7 pages. https://doi.org/10.1145/3321335.3324939

[10] Euan Freeman, Julie Williamson, Sriram Subramanian, and Stephen Brewster 2018. Point-and-Shake: Selecting from Levitating Object Displays. In Proceedings of the 2018 CHI Conference on Human Factors in Computing Systems (Montreal QC, Canada) (CHI '18). Association for Computing Machinery, New York, NY, USA, Article 18, 10 pages. https://doi.org/10.1145/3173574.3173592

[11] Markus Funk. 2018. Human-Drone Interaction: Let's Get Ready for Flying User Interfaces! Interactions 25, 3 (April 2018), 78-81. https://doi.org/10.1145/3194317

[12] Takuro Furumoto, Keisuke Hasegawa, Yasutoshi Makino, and Hiroyuki Shinoda. 2019. Three-Dimensional Manipulation of a Spherical Object Using Ultrasound Plane Waves. IEEE Robotics and Automation Letters 4, 1 (2019), 81-88.

[13] Tatsuki Fushimi, Asier Marzo, Bruce W. Drinkwater, and Thomas L. Hill. 2019 Acoustophoretic volumetric displays using a fast-moving levitated particle. Applied Physics Letters 115, 6 (2019), 064101. https://doi.org/10.1063/1.5113467 arXiv:https://doi.org/10.1063/1.5113467

[14] Antonio Gomes, Calvin Rubens, Sean Braley, and Roel Vertegaal. 2016. BitDrones: Towards Using 3D Nanocopter Displays as Interactive Self-Levitating Programmable Matter. In Proceedings of the 2016 CHI Conference on Human Factors in Computing Systems (San Jose, California, USA) (CHI '16). Association for Computing Machinery, New York, NY, USA, 770-780. https://doi.org/10.1145/ 2858036.2858519

[15] Keisuke Hasegawa and Hiroyuki Shinoda. 2018. Aerial Vibrotactile Display Based on Multiunit Ultrasound Phased Array. IEEE Transactions on Haptics 11, 3 (2018), 367-377. https://doi.org/10.1109/TOH.2018.2799220

[16] Ryuji Hirayama, Diego Martinez Plasencia, Nobuyuki Masuda, and Sriram Subramanian. 2019. A volumetric display for visual, tactile and audio presentation using acoustic trapping. Nature 575, 7782 (2019), 320-323. https: //doi.org/10.1038/s41586-019-1739-5

[17] T Hoshi, M Takahashi, T Iwamoto, and H Shinoda. 2010. Noncontact Tactile Display Based on Radiation Pressure of Airborne Ultrasound. IEEE Transactions on Haptics 3, 3 (2010), 155-165. https://doi.org/10.1109/TOH.2010.4

[18] Seki Inoue, Yasutoshi Makino, and Hiroyuki Shinoda. 2016. Scalable Architecture for Airborne Ultrasound Tactile Display. In AsiaHaptics (Lecture Notes in Electrical Engineering). Springer, Singapure, 2035-2040. https://doi.org/10.1007/978-98110-4157-0_17

[19] Pascal Knierim, Thomas Kosch, Alexander Achberger, and Markus Funk. 2018. Flyables: Exploring 3D Interaction Spaces for Levitating Tangibles. In Proceedings of the Twelfth International Conference on Tangible, Embedded, and Embodied Interaction (Stockholm, Sweden) (TEI '18). Association for Computing Machinery, New York, NY, USA, 329-336. https://doi.org/10.1145/3173225.3173273

[20] Joseph La Delfa, Mehmet Aydin Baytas, Rakesh Patibanda, Hazel Ngari, Rohit Ashok Khot, and Florian 'Floyd' Mueller. 2020. Drone Chi: Somaesthetic Human-Drone Interaction. In Proceedings of the 2020 CHI Conference on Human Factors in Computing Systems (Honolulu, HI, USA) (CHI '20). Association for Computing Machinery, New York, NY, USA, 1-13. https://doi.org/10.1145/3313831. 3376786

[21] P. J. J. Lamoré, H. Muijser, and C. J. Keemink. 1986. Envelope detection of amplitude-modulated high-frequency sinusoidal signals by skin mechanoreceptors. The Fournal of the Acoustical Society of America 79, 4 (1986), 1082-1085. https://doi.org/10.1121/1.393380 arXiv:https://doi.org/10.1121/1.393380

[22] Jinha Lee, Rehmi Post, and Hiroshi Ishii. 2011. ZeroN: Mid-air Tangible Interaction Enabled by Computer Controlled Magnetic Levitation. In Proceedings of the 24th Annual ACM Symposium on User Interface Software and Technology (UIST '11) ACM, New York, NY, USA, 327-336. https://doi.org/10.1145/2047196.2047239

[23] Marjorie R Leek. 2001. Adaptive procedures in psychophysical research. Perception \& psychophysics 63, 8 (2001), 1279-1292.

[24] Benjamin Long, Sue Ann Seah, Tom Carter, and Sriram Subramanian. 2014. Ren dering volumetric haptic shapes in mid-air using ultrasound. ACM Transactions on Graphics 33, 6 (2014), 1-10. https://doi.org/10.1145/2661229.2661257

[25] Mark T Marshall, Tom Carter, Jason Alexander, and Sriram Subramanian. 2012 Ultra-Tangibles : Creating Movable Tangible Objects on Interactive Tables. Proc. CHI 2012 (2012), 2185-2188. https://doi.org/10.1145/2208276.2208370

[26] Rafael Morales, Asier Marzo, Sriram Subramanian, and Diego Martínez. 2019 LeviProps: Animating Levitated Optimized Fabric Structures Using Holographic Acoustic Tweezers. In Proceedings of the 32nd Annual ACM Symposium on User Interface Software and Technology (New Orleans, LA, USA) (UIST '19). Association for Computing Machinery, New York, NY, USA, 651-661. https://doi.org/10. 1145/3332165.3347882

[27] Tao Morisaki, Ryoma Mori, Ryosuke Mori, Yasutoshi Makino, Yuta Itoh, Yuji Yamakawa, and Hiroyuki Shinoda. 2019. Hopping-Pong: Changing Trajectory of Moving Object Using Computational Ultrasound Force. In Proceedings of the 2019 ACM International Conference on Interactive Surfaces and Spaces (Daejeon, Republic of Korea) (ISS '19). Association for Computing Machinery, New York, NY, USA, 123-133. https://doi.org/10.1145/3343055.3359701

[28] Kei Nitta, Keita Higuchi, and Jun Rekimoto. 2014. HoverBall: Augmented Sports with a Flying Ball. In Proceedings of the 5th Augmented Human International Conference (Kobe, Japan) (AH '14). Association for Computing Machinery, New York, NY, USA, Article 13, 4 pages. https://doi.org/10.1145/2582051.2582064

[29] Mohd Adili Norasikin, Diego Martinez Plasencia, Spyros Polychronopoulos, Gianluca Memoli, Yutaka Tokuda, and Sriram Subramanian. 2018. SoundBender: Dynamic Acoustic Control Behind Obstacles. In Proceedings of the 31st Annual ACM Symposium on User Interface Software and Technology (Berlin, Germany) (UIST '18). Association for Computing Machinery, New York, NY, USA, 247-259. https://doi.org/10.1145/3242587.3242590

[30] Yoichi Ochiai, Takayuki Hoshi, and Jun Rekimoto. 2014. Pixie Dust: Graphics Generated by Levitated and Animated Objects in Computational Acoustic-potential Field. ACM Trans. Graph. 33, 4 (2014), 85:1--85:13. https://doi.org/10.1145/ 2601097.2601118

[31] Themis Omirou, Asier Marzo, Sue Ann Seah, and Sriram Subramanian. 2015. LeviPath. Proceedings of the 33rd Annual ACM Conference on Human Factors in Computing Systems - CHI '15 (2015), 309-312. https://doi.org/10.1145/2702123. 2702333

[32] T. Omirou, A. M. Perez, S. Subramanian, and A. Roudaut. 2016. Floating charts: Data plotting using free-floating acoustically levitated representations. In 2016 IEEE Symposium on 3D User Interfaces (3DUI). 187-190. https://doi.org/10.1109/ 3DUI.2016.7460051

[33] R. B. Rusu and S. Cousins. 2011. 3D is here: Point Cloud Library (PCL). In 2011 IEEE International Conference on Robotics and Automation. 1-4. https: //doi.org/10.1109/ICRA.2011.5980567

[34] K. Suita, Y. Yamada, N. Tsuchida, K. Imai, H. Ikeda, and N. Sugimoto, 1995. A failure-to-safety "Kyozon" system with simple contact detection and stop capabilities for safe human-autonomous robot coexistence. In Proceedings of 1995 IEEE International Conference on Robotics and Automation, Vol. 3. 3089-3096 vol.3.

[35] Ivan E. Sutherland. 1965. The Ultimate Display. In Proceedings of the IFIP Congress. 506-508

[36] S. Suzuki, S. Inoue, M. Fujiwara, Y. Makino, and H. Shinoda. 2021. AUTD3: Scalable Airborne Ultrasound Tactile Display. IEEE Transactions on Haptics (2021), 1-1. https://doi.org/10.1109/TOH.2021.3069976

[37] Michael J. Swain and Dana H. Ballard. 1992. Indexing via Color Histograms. In Active Perception and Robot Vision, Arun K. Sood and Harry Wechsler (Eds.). Springer Berlin Heidelberg, Berlin, Heidelberg, 261-273.

[38] Tommaso Toffoli and Norman Margolus. 1991. Programmable matter: concepts and realization. Physica. D, Nonlinear phenomena 47, 1-2 (1991), 263-272.

[39] Brygg Ullmer and Hiroshi Ishii. 1997. The MetaDESK: Models and Prototypes for Tangible User Interfaces. In Proceedings of the 10th Annual ACM Symposium on User Interface Software and Technology (Banff, Alberta, Canada) (UIST '97). Association for Computing Machinery, New York, NY, USA, 223-232. https: //doi.org/10.1145/263407.263551

[40] F.M. White. 1991. Viscous Fluid Flow. McGraw-Hill. 174-176 pages. https: //books.google.co.jp/books?id=RBA3AgAACAAJ

[41] Wataru Yamada, Hiroyuki Manabe, and Daizo Ikeda. 2019. ZeRONE: Safety Drone with Blade-Free Propulsion. In Proceedings of the 2019 CHI Conference on Human Factors in Computing Systems (Glasgow, Scotland Uk) (CHI '19). ACM, New York, NY, USA, Article 365, 8 pages. https://doi.org/10.1145/3290605.3300595

[42] Wataru Yamada, Kazuhiro Yamada, Hiroyuki Manabe, and Daizo Ikeda. 2017. iSphere: Self-Luminous Spherical Drone Display. Proceedings of the 30th Annual ACM Symposium on User Interface Software and Technology - UIST '17 (2017). https://doi.org/10.1145/3126594.3126631

[43] Toshiya Yui and Tomoko Hashida. 2016. Floatio: Floating Tangible User Interface Based on Animacy Perception. In Proceedings of the 29th Annual Symposium on User Interface Software and Technology (Tokyo, Japan) (UIST '16 Adjunct). Association for Computing Machinery, New York, NY, USA, 43-45. https://doi. org/10.1145/2984751.2985699

[44] Xujing Zhang, Sean Braley, Calvin Rubens, Timothy Merritt, and Roel Vertegaal. 2019. LightBee: A Self-Levitating Light Field Display for Hologrammatic Telepresence. In Proceedings of the 2019 CHI Conference on Human Factors in Computing Systems (CHI '19). ACM, New York, NY, USA, 12:1--12:10. https://doi.org/10.1145/3290605.3300242 